\title{
ZNF226 is over-expressed in brain metastatic breast cancer.
}

Shahan Mamoor

East Islip, NY USA ${ }^{1}$

1shahanmamoor@gmail.com

Metastasis to the brain is a clinical problem in patients with breast cancer ${ }^{1-3}$. We mined published microarray data 4,5 to compare primary and metastatic tumor transcriptomes to discover genes associated with brain metastasis in patients with metastatic breast cancer. We found that the zinc finger protein 226, encoded by ZNF226 was among the genes whose expression was most different in the brain metastases of patients with brain metastatic breast cancer as compared to primary tumors of the breast. ZNF226 may be relevant to processes underlying metastasis of primary tumor-derived cancer cells to the brain in humans with metastatic breast cancer.

Keywords: breast cancer, brain metastases, central nervous system metastases, ZNF226, systems biology of breast cancer, targeted therapeutics in breast cancer. 
One report described a 34\% incidence of central nervous system metastases in trastuzumabtreated patients with breast cancer ${ }^{2}$. This alarmingly high frequency of CNS metastasis events demands an enhanced understanding of the transcriptional makeup of brain metastatic tissues to support identification of therapeutic targets, whether they are treatment related or not. We performed a global comparative analysis of primary and metastatic tumors in patients with brain metastatic breast cancer ${ }^{4,5}$. We discovered significant differential and increased expression of ZNF226 in brain metastatic tissues of patients with metastatic breast cancer.

\section{Methods}

We used datasets GSE1259894 and GSE526045 for this global differential gene expression analysis of brain metastatic breast cancer in conjunction with GEO2R. GSE125989 was generated using Affymetrix Human Genome U133A 2.0 Array technology with $n=16$ primary tumors from patients with breast cancer and $n=16$ brain metastases from patients with brain metastatic breast cancer. GSE52604 was generated using Whole Human Genome Microarray 4x44K G4112F technology with $n=10$ normal breast tissues and $\mathrm{n}=35$ brain metastases from patients with breast cancer, analysis performed using platform GPL6480. The Benjamini and Hochberg method of $p$-value adjustment was used for ranking of differential expression but raw $p$-values were used to assess statistical significance of global differential expression. Log-transformation of data was auto-detected, and the NCBI generated category of platform annotation was used. A statistical test was performed to evaluate whether ZNF226 gene expression was significantly between primary breast tumors and brain metastases in humans with breast cancer using a two-tailed, unpaired t-test with Welch's correction. We used PRISM for all statistical analyses of differential gene expression in human breast cancer (Version 8.4.0)(455).

\section{Results}

We performed global comparative transcriptome analysis of the primary and metastatic tumor tissues of patients with brain metastatic breast cancer using published microarray data ${ }^{4,5}$ to describe the transcriptional landscape of brain metastasis in human breast cancer in an unbiased fashion and for the discovery of novel therapeutic targets.

\section{ZNF226 is differentially expressed in the brain metastases of patients with brain metastatic breast} cancer.

We identified ZNF226 as among the genes whose expression was most significantly different in the brain metastases of patients with brain metastatic breast cancer ${ }^{4}$ (Table 1). When sorting each of the genes expressed in brain metastases based on significance of difference as compared to the primary tumors of patients with brain metastatic breast cancer, ZNF226 ranked 153 out of 22297 total transcripts (Table 1), equating to $99.3 \%$ differential expression. Differential expression of ZNF226 in the brain metastases of patients with brain metastatic breast cancer was statistically significant (Table 1; $p=8.92 \mathrm{E}-04$ ). more challenging than when cross-validating across organs and sorted cell populations due to higher levels of heterogeneity in tumors, and differing methods of tumor sampling between laboratories. Thus, we asked whether we could validate differential expression of ZNF226 when comparing brain metastases from patients with breast cancer to normal breast tissue as opposed to primary tumors of the breast. Comparison of global gene expression profiles of 10 normal breast tissues to 35 brain metastases 5 revealed that ZNF226 was among the genes whose expression was most significantly different transcriptome-wide between the breast and brain metastases in patients with breast cancer (Table 2). 
When sorting each of the genes expressed in brain metastases based on significance of difference in expression between brain metastases and normal breast tissues, ZNF226 ranked 6513 out of 41093 total transcripts (Table 2), equating to $84.2 \%$ differential expression. Differential expression of ZNF226 in brain metastases from patients with brain metastatic breast cancer in this dataset was statistically significant (Table $2 ; p=7.19 \mathrm{E}-04)$.

5

\section{ZNF226 is expressed at significantly higher levels in the brain metastases of patients with brain} metastatic breast cancer.

We obtained exact mRNA expression levels for ZNF226, in primary tumors of the breast and in brain metastasis of patients with brain metastatic breast cancer to determine magnitude and direction of change in ZNF226 expression in brain metastatic tissues. ZNF226 was expressed at higher levels in the brain metastases of patients with breast cancer as compared to primary tumors of the breast, and this difference was statistically significant (Figure $1 ; p=0.0007$ ). We calculated a mean fold change of $1.47 \pm$ 0.19 in ZNF226 expression when comparing primary tumors of the breast to brain metastatic tissues in patients with breast cancer (Table 1).

Thus, by mining published microarray data 4,5 in an unbiased and systematic fashion, we identified ZNF226 as among the genes whose expression was most different in the brain metastases of patients with breast cancer both when compared to primary tumors of the breast and to the normal breast; ZNF226 was expressed at significantly higher levels in brain metastases as compared to primary tumors of the breast.

\section{Discussion}

ZNF226 belongs to the $\mathrm{C} 2 \mathrm{H} 2 \mathrm{ZNF}$ transcription factors, also known as Kruppel-type zinc fingercontaining (ZNF) proteins, which share in common a domain with the ability to repress transcription known as Kruppel-associated box, or KRAB ${ }^{6}$. ZNF226 contains KRAB-A and KRAB-B domains at its amino-terminus, $19 \mathrm{ZNF}$ repeats, and a spacer region separating that KRAB-A/B domains from the carboxy-terminal ZNF repeats ${ }^{6}$. In general, ZNF repeats of Kruppel-type zinc finger transcription factors function as a DNA-binding domain, while the KRAB domains function in transcriptional repression 7 . ZNF226 is described to possess ubiquitous expression and the ability to repress RNA Polymerase II ${ }^{7}$. A study performing whole genome sequencing of 27 hepatocellular carcinomas found mutation of ZNF226 in 3 out of 27 cases $^{8}$. Thus, ZNF226 is a Kruppel-type zinc finger transcription factor with the ability to repress RNA Pol II and the gene encoding ZNF226 is mutated in a subset of human hepatocellular carcinomas.

We report here that ZNF226 is among the genes whose expression is most different in the brain metastases of patients with brain metastatic breast cancer. Understand in greater detail the transcriptional targets of ZNF226, and how gene expression is most significantly altered in isolated tumor cells from primary and metastatic tumors after expression of ZNF226 may provide insights into molecular mechanisms underlying metastasis to the brain in patients with metastatic breast cancer.

PAGE 3 


\section{References}

1. Lin, N.U., Amiri-Kordestani, L., Palmieri, D., Liewehr, D.J. and Steeg, P.S., 2013. CNS metastases in breast cancer: old challenge, new frontiers.

2. Bendell, J.C., Domchek, S.M., Burstein, H.J., Harris, L., Younger, J., Kuter, I., Bunnell, C., Rue, M., Gelman, R. and Winer, E., 2003. Central nervous system metastases in women who receive trastuzumab-based therapy for metastatic breast carcinoma. Cancer, 97(12), pp.2972-2977.

3. Tsukada, Y., Fouad, A., Pickren, J.W. and Lane, W.W., 1983. Central nervous system metastasis from breast carcinoma autopsy study. Cancer, 52(12), pp.2349-2354.

4. Iwamoto, T., Niikura, N., Ogiya, R., Yasojima, H., Watanabe, K.I., Kanbayashi, C., Tsuneizumi, M., Matsui, A., Fujisawa, T., Iwasa, T. and Shien, T., 2019. Distinct gene expression profiles between primary breast cancers and brain metastases from pair-matched samples. Scientific reports, 9(1), pp. $1-8$.

5. Salhia, B., Kiefer, J., Ross, J.T., Metapally, R., Martinez, R.A., Johnson, K.N., DiPerna, D.M., Paquette, K.M., Jung, S., Nasser, S. and Wallstrom, G., 2014. Integrated genomic and epigenomic analysis of breast cancer brain metastasis. PloS one, 9(1), p.e85448.

6. Shannon, M., Hamilton, A.T., Gordon, L., Branscomb, E. and Stubbs, L., 2003. Differential expansion of zinc-finger transcription factor loci in homologous human and mouse gene clusters. Genome research, 13(6a), pp.1097-1110.

7. Urrutia, R., 2003. KRAB-containing zinc-finger repressor proteins. Genome biology, 4(10), p.231.

8. Fujimoto, A., Totoki, Y., Abe, T., Boroevich, K.A., Hosoda, F., Nguyen, H.H., Aoki, M., Hosono, N., Kubo, M., Miya, F. and Arai, Y., 2012. Whole-genome sequencing of liver cancers identifies etiological influences on mutation patterns and recurrent mutations in chromatin regulators. Nature genetics, 44(7), pp.760-764.

PAGE 4 


\section{Table 1: ZNF226 is differentially expressed in brain metastases in brain metastatic breast cancer.}

Rank of differential expression, probe ID, $p$-value with respect to differential expression, $t$, a moderated tstatistic, B, the log-odds of differential expression between the two groups compared, fold change (FC) of ZNF226 expression between the brain metastases of patients with breast cancer and primary tumors of the breast, gene and gene name are listed in this chart. 
PAGE 6 


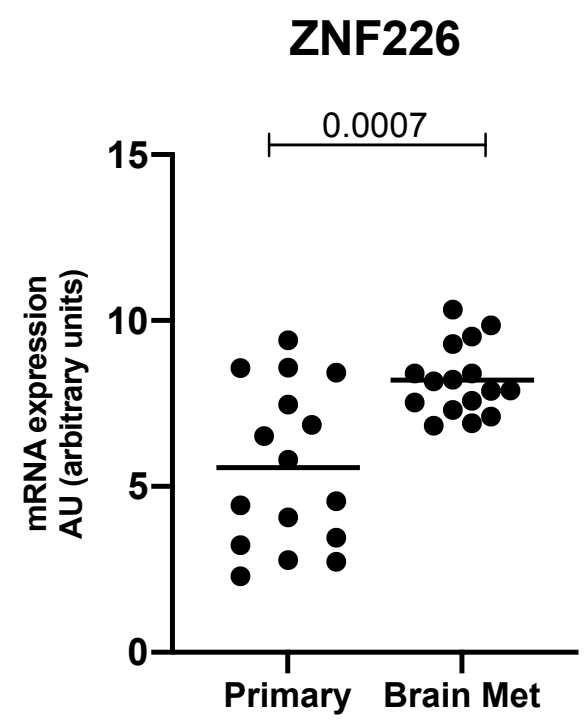

Figure 1: ZNF226 is expressed at significantly higher levels in the brain metastases of patients with brain metastatic breast cancer.

The mRNA expression level of ZNF226 in primary tumors of the breast (left) and in the brain metastases of brain metastatic breast cancer patients (right) is graphically represented here with mean ZNF226 levels marked and the result of a statistical test evaluating difference in mRNA expression level between brain metastases and primary tumors of the breast, a $p$-value, listed above. 